\title{
CARACTERIZAÇÃO QUÍMICA E MICROESTRUTURAL DE HASTES FEMORAIS MODULARES FABRICADAS EM AÇO INOXIDÁVEL ABNT NBR ISO 5832-9*
}

\section{Resumo}

O objetivo do presente trabalho foi avaliar e comparar a composição química e microestrutura de dois fabricantes (nacional e importado) de haste femoral fabricados em aço inoxidável austenítico ABNT NBR ISO 5832-9. As análises químicas dos principais elementos foram realizadas utilizando espectrometria de emissão ótica por descarga luminescente. A microestrutura das hastes foi caracterizada por microscopia óptica, microscopia eletrônica de varredura e difração de raios X. Medidas de dureza Vickers também foram realizadas neste estudo comparativo. As hastes nacionais e importadas exibiram pequena quantidade de partículas de nitreto (fase Z) dispersas na matriz austenítica e tamanhos de grão similares da austenita. Por outro lado, o aumento de $32 \%$ observado nos resultados de dureza da haste importada em relação à haste nacional foi atribuído às diferenças de composição química entre estas hastes. Foram identificadas microinclusões ricas em Al nas hastes importadas e microinclusões ricas em $\mathrm{Mg}$ nas hastes nacionais. Embora tenham sido observadas algumas diferenças entre as hastes nacionais e importadas, ambas atenderam os critérios de composição e microestrutura da norma ABNT NBR ISO 5832-9.

Palavras-chave: Implante de quadril; Haste femoral; Aço inoxidável; Microestrutura.

\section{CHEMICAL AND MICROSTRUCTURAL CHARACTERIZATION OF MODULAR FEMORAL STEMS MADE OF STAINLESS STEEL ISO 5832-9.}

\footnotetext{
Abstract

The aim of this study was to evaluate and compare the chemical composition and microstructure of two models of femoral stem (national and imported) manufactured from ISO 5832-9 austenitic stainless steel. Chemical analyses of the key elements were performed by glow discharge optical emission spectrometry. The microstructure of the stems was characterized by optical microscopy, scanning electron microscopy and X-ray diffraction. Vickers hardness measurements were also performed in this comparative study. The national and imported stems exhibited small amount of nitride particles (Z-phase) dispersed in the austenitic matrix and similar grain sizes of austenite. On the other hand, the hardness was $32 \%$ higher in the imported stem, which was attributed to the differences in composition between the stems. Al-rich and Mg-rich inclusions were identified in the imported and national stems, respectively. Although some differences have been observed between national and imported stems, both met the criteria of composition and microstructure of the ISO 5832-9 standard.

Keywords: Hip implant; Femoral stem; Stainless steel; Microstructure.

1 Engenharia de Materiais, D.Sc., pesquisadora, LEBm, HU, Universidade Federal de Santa Catarina (UFSC), Florianópolis, SC, Brasil.

2 Engenheiro de Materiais, D.Sc., tecnologista, DEMP, LACPM, Instituto Nacional de Tecnologia (INT), Rio de Janeiro, RJ, Brasil.

Engenheira Metalúrgica, D.Sc., tecnologista, DEMP, LACPM, INT, Rio de Janeiro, RJ, Brasil. Engenheiro Mecânico, D.Sc., tecnologista, DEMP, LACPM, INT, Rio de Janeiro, RJ, Brasil. Engenheiro Metalúrgico, M.Sc., tecnologista, DEMP, LACPM, INT, Rio de Janeiro, RJ, Brasil. Engenharia de Materiais, graduando, bolsista, LEBm, HU, UFSC, Florianópolis, SC, Brasil. Engenheiro Mecânico, D.Sc., professor, LEBm, HU, UFSC, Florianópolis, SC, Brasil.
} 


\section{INTRODUÇÃO}

Os implantes de quadril de aço inoxidável são amplamente utilizados no Brasil pelo Sistema Único de Saúde (SUS), embora no mercado nacional existam implantes fabricados com materiais metálicos melhores em termos de biocompatibilidade e resistência mecânica, tais como o titânio e suas ligas e a liga Co-Cr-Mo. Isto se deve à combinação única de baixo custo, boas propriedades mecânicas, boa resistência à corrosão e razoável biocompatibilidade encontrada no aço.

O aço inoxidável austenítico de especificação ASTM F138 (ou ABNT NBR ISO 58321) é o aço mais utilizado em implantes ortopédicos e, a necessidade de melhorar as suas propriedades levou ao desenvolvimento do aço inoxidável austenítico de alto teor de nitrogênio de especificação ABNT NBR ISO 5832-9 (Implantes para cirurgia - materiais metálicos - Parte 9: Aço inoxidável conformado de alto nitrogênio), que é um aço indicado para aplicações envolvendo carregamentos severos e longos períodos de permanência no interior do corpo humano [1,2]. O nitrogênio é um elemento crucial no aço porque estabiliza a austenita, aumenta a resistência mecânica e previne a corrosão localizada [3]. Pequenas quantidades de nitrogênio já são suficientes para promover variações significativas nas propriedades mecânicas e de corrosão [4]. O aumento de resistência mecânica é ocasionado pelo endurecimento por solução sólida, formação de precipitados da fase $Z$ (nitreto rico em $\mathrm{Nb}$ e $\mathrm{Cr}$, contendo também $\mathrm{Fe}$ e $\mathrm{Mo}$ ) e refino de grão [1]. A maior resistência à corrosão é atribuída ao aumento da estabilidade do filme passivo, favorecida pela presença do nitrogênio em solução sólida na austenita [1]. Grabke [5] sugeriu que o nitrogênio carregado negativamente logo abaixo do filme passivo pode inibir a corrosão localizada, suprimindo ou retardando a adsorção de íons agressivos $\mathrm{Cl}^{-}$no filme passivo.

A microestrutura e as propriedades finais dos aços para aplicações médicas são bastante dependentes da evolução microestrutural durante o processo de fabricação do aço e durante o forjamento a quente do implante [6]. Devido à maior complexidade da microestrutura do aço ABNT NBR ISO 5832-9 em relação ao ASTM F138 é essencial caracterizar a sua microestrutura para avaliar o efeito do processo de fabricação nas fases formadas e nas propriedades finais do implante. Por exemplo, as partículas da fase $Z$ que precipitam durante a fabricação do aço de alto nitrogênio têm tamanhos maiores e se apresentam na forma alongada (placas), enquanto que as partículas que se formam durante o forjamento a quente são menores e tem a forma mais arredondada. As partículas mais grosseiras podem atuar como concentradores de tensão e, consequentemente, ter importante participação na iniciação e propagação de trincas de fadiga [1]. Giordano et al. [4] sugerem que adições de $\mathrm{Nb}$ também devem ser bem controladas para evitar a precipitação excessiva de fase $Z$, pois isto envolveria a remoção de nitrogênio da matriz ao redor das partículas, tornando essas regiões mais susceptíveis à corrosão. Portanto, para se alcançar as propriedades requeridas pela norma, a quantidade e a morfologia das partículas da fase $\mathrm{Z}$ e a estrutura de grãos da austenita devem ser bem controlados durante o processo de fabricação.

Estudos realizados nos Estados Unidos mostraram que 10\% dos implantes removidos de pacientes por apresentarem problemas durante o uso não atendiam aos critérios da norma ASTM [7]. No Brasil ainda não há um acompanhamento efetivo dos implantes disponíveis no mercado em relação ao atendimento às normas vigentes. 
Os implantes de quadril modulares têm como principais vantagens a flexibilidade para aplicações em diversas anatomias e a possibilidade de combinação de diferentes materiais. Estes implantes possuem pelo menos duas partes modulares: haste e cabeça femorais.

O presente estudo tem como objetivo a caracterização e comparação da composição química e microestrutura de duas hastes femorais para implantes de quadril modulares: um fabricante nacional e um importado, ambos fabricados em aço inoxidável austenítico de alto teor de nitrogênio ABNT NBR ISO 5832-9.

\section{MATERIAIS E MÉTODOS}

No presente trabalho foram estudados dois fabricantes de hastes femorais de implantes de quadril modulares, um fabricado no Brasil e outro fabricado no exterior (Figura 1), ambos produzidos em aço inoxidável austenítico ABNT NBR ISO 5832-9 e indicados para artroplastia usando cimento ósseo (superfície polida). Foram caracterizadas três hastes novas e idênticas de cada fabricante $(n=3)$. A Tabela 1 apresenta outras informações das próteses estudadas.
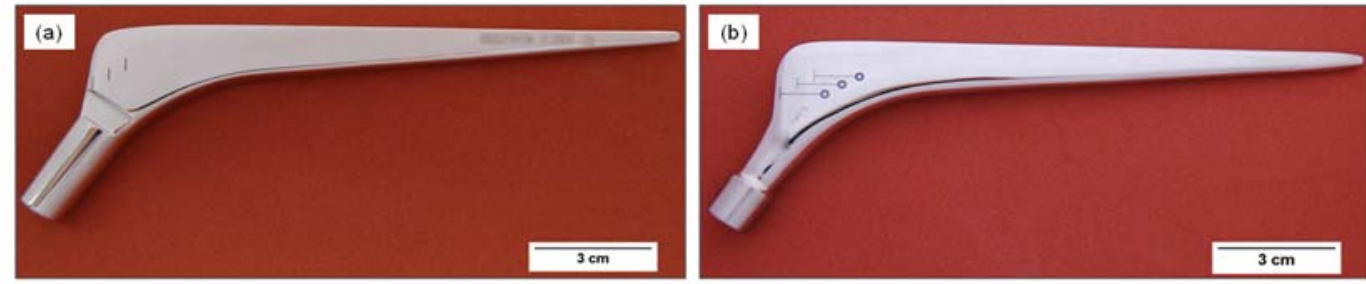

Figura 1. Haste femoral (a) nacional e (b) importada como recebida.

Tabela 1. Características técnicas das hastes femorais utilizadas neste estudo.

\begin{tabular}{ccc}
\hline Haste femoral & Nacional & Importada \\
\hline Tipo & Cimentada & Cimentada \\
Acabamento superficial & Polida & Polida \\
Comprimento da haste $(\mathrm{mm})$ & 150 & 150 \\
Offset $(\mathrm{mm})$ & 37,5 & 37,5 \\
Material & Aço inox austenítico de alto $\mathrm{N}$ & Aço inox austenítico de alto N \\
Especificação & ABNT NBR ISO 5832-9 & ABNT NBR ISO 5832-9 \\
Processo de fabricação & Forjada a Quente & Forjada a Quente \\
\hline
\end{tabular}

\subsection{Análise química das hastes}

As análises químicas das hastes foram realizadas utilizando as técnicas de combustão direta da amostra com detecção por infravermelho (C e S), fusão da amostra em ambiente de gás inerte com detecção por condutividade térmica $(\mathrm{N})$ e espectrometria de emissão ótica por descarga luminescente (demais elementos).

\subsection{Corte e preparação metalográfica}

Na Figura 2 estão indicadas as quatro regiões da haste que foram escolhidas para a caracterização microestrutural e medidas de dureza. Estas regiões foram representadas por amostras nomeadas na sequência de $A$ até $D$, sendo analisada a seção transversal $(t)$ da haste em cada região. Na região $C$ (meio da haste) também foi analisada a seção longitudinal $(\ell)$ para a determinação do teor de inclusões. As 
linhas tracejadas indicam as posições dos cortes transversais e a área hachurada indica o corte longitudinal.

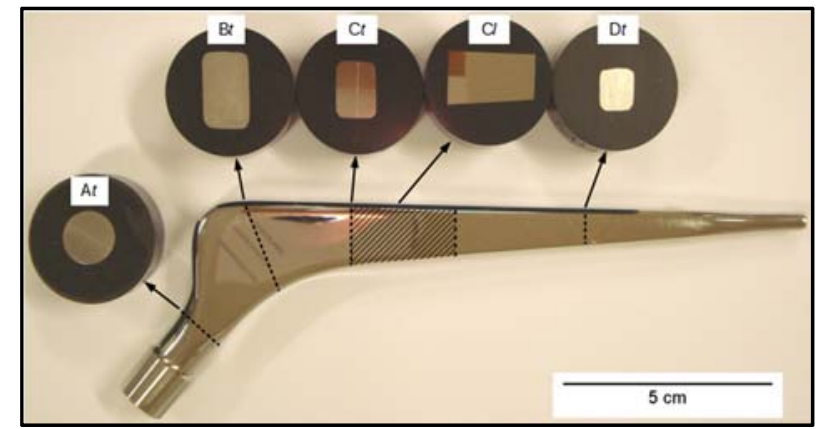

Figura 2. Esquema de corte de amostras das hastes para a caracterização. le importada.

\begin{tabular}{cccc}
\hline Elemento & $\begin{array}{c}\text { ABNT NBR ISO } \\
\mathbf{5 8 3 2 - 9} \text { (\% em peso) }\end{array}$ & $\begin{array}{c}\text { Haste Nacional } \\
\text { (\% em peso) }\end{array}$ & $\begin{array}{c}\text { Haste Importada } \\
\text { (\% em peso) }\end{array}$ \\
\hline $\mathrm{C}$ & 0,08 máx. & $0,043 \pm 0,007$ & $0,038 \pm 0,004$ \\
$\mathrm{Si}$ & 0,75 máx. & $0,14 \pm 0,03$ & $0,440 \pm 0,002$ \\
$\mathrm{Mn}$ & $2-4,25$ & $3,83 \pm 0,02$ & $4,01 \pm 0,05$ \\
$\mathrm{Ni}$ & $9,0-11,0$ & $9,8 \pm 0,5$ & $9,3 \pm 0,4$ \\
$\mathrm{Cr}$ & $19,5-22,0$ & $19,5 \pm 0,4$ & $19,5 \pm 0,4$ \\
$\mathrm{Mo}$ & $2,0-3,0$ & $2,3 \pm 0,1$ & $2,0 \pm 0,1$ \\
$\mathrm{Nb}$ & $0,25-0,8$ & $0,33 \pm 0,06$ & $0,33 \pm 0,06$ \\
$\mathrm{~S}$ & 0,010 máx. & $<0,002$ & $<0,001$ \\
$\mathrm{P}$ & 0,025 máx. & $0,009 \pm 0,002$ & $0,020 \pm 0,001$ \\
$\mathrm{Cu}$ & 0,25 máx. & $0,037 \pm 0,006$ & $0,070 \pm 0,007$ \\
$\mathrm{~N}$ & $0,25-0,5$ & $0,33 \pm 0,02$ & $0,417 \pm 0,006$ \\
$\mathrm{Fe}$ & Balanço & $63,7 \pm 0,2$ & $63,8 \pm 0,6$ \\
\hline
\end{tabular}

\subsection{Fases presentes}

As Figuras 3(a) e 3(b) mostram as microestruturas da seção transversal $\mathrm{Ct}$ das hastes femorais nacional e importada, respectivamente, que revelaram a existência da matriz austenítica com precipitados dispersos de fase $Z$ (pontos claros) nas duas amostras. A fase $Z$ é um nitreto de $\mathrm{Nb}$ e $\mathrm{Cr}$ inerente ao aço de alto teor de nitrogênio. No aço ABNT NBR ISO 5832-9, a fase $Z$ contém também o Mo e o $\mathrm{Fe}$ ocupando parcialmente as posições atômicas do $\mathrm{Nb}$ e do $\mathrm{Cr}$, respectivamente. A fórmula da fase $\mathrm{Z}$ é aproximadamente $\left(\mathrm{Cr}_{0,8} \mathrm{Fe}_{0,2}\right)\left(\mathrm{Nb}_{0,9} \mathrm{Mo}_{0,1}\right) \mathrm{N}$, podendo apresentar outras proporções entre os elementos de acordo com a composição do aço [8]. Como a fase $Z$ é um composto frágil, as partículas mais grosseiras atuam como concentradores de tensão, podendo ter importante participação na iniciação e propagação de trincas de fadiga, seja pela separação da interface matriz-partícula ou pela fratura da própria partícula, que é um processo propenso a ocorrer quando o aço é submetido a cargas cíclicas [1,2]. Além disso, quando o material esta imerso em um meio agressivo, a descontinuidade geométrica criada pela fratura da partícula favorece a ocorrência de corrosão por frestas, o que também contribui para a iniciação e propagação de trincas de fadiga [1,2]. Portanto, a quantidade e morfologia das partículas da fase $Z$ são fatores importantes que devem ser bem controlados durante o processo de fabricação da matéria-prima e do implante. 

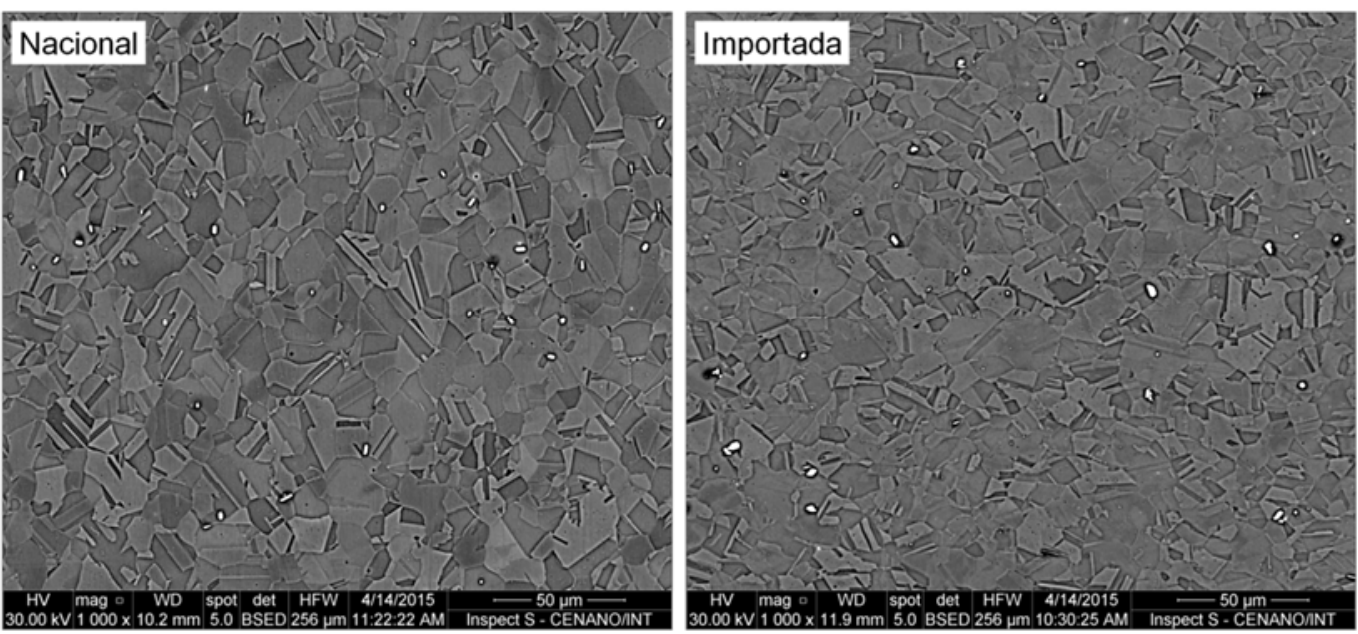

Figura 3. Imagens de MEV (elétrons retroespalhados) da parte central da seção transversal $\mathrm{C} t$ das hastes femorais nacional e importada.

A análise por difração de raios $X$ confirmou a presença da fase $Z$ e da austenita (fase $\gamma$ ) em ambas as hastes femorais. Os difratogramas experimentais e a identificação das fases presentes pela comparação com os picos simulados são mostrados nas Figuras 4 e 5 . As estruturas cristalinas das fases estão inseridas na Figura 4 , mostrando que a fase $Z$ possui estrutura tetragonal, constituída de três sub-redes ocupadas pelos átomos de $\mathrm{Nb}$ (ou Mo), $\mathrm{Cr}$ (ou Fe) e N. A microestrutura da haste importada mostrando a fase $\mathrm{Z}$ ampliada pode ser vista no difratograma da Figura 5.

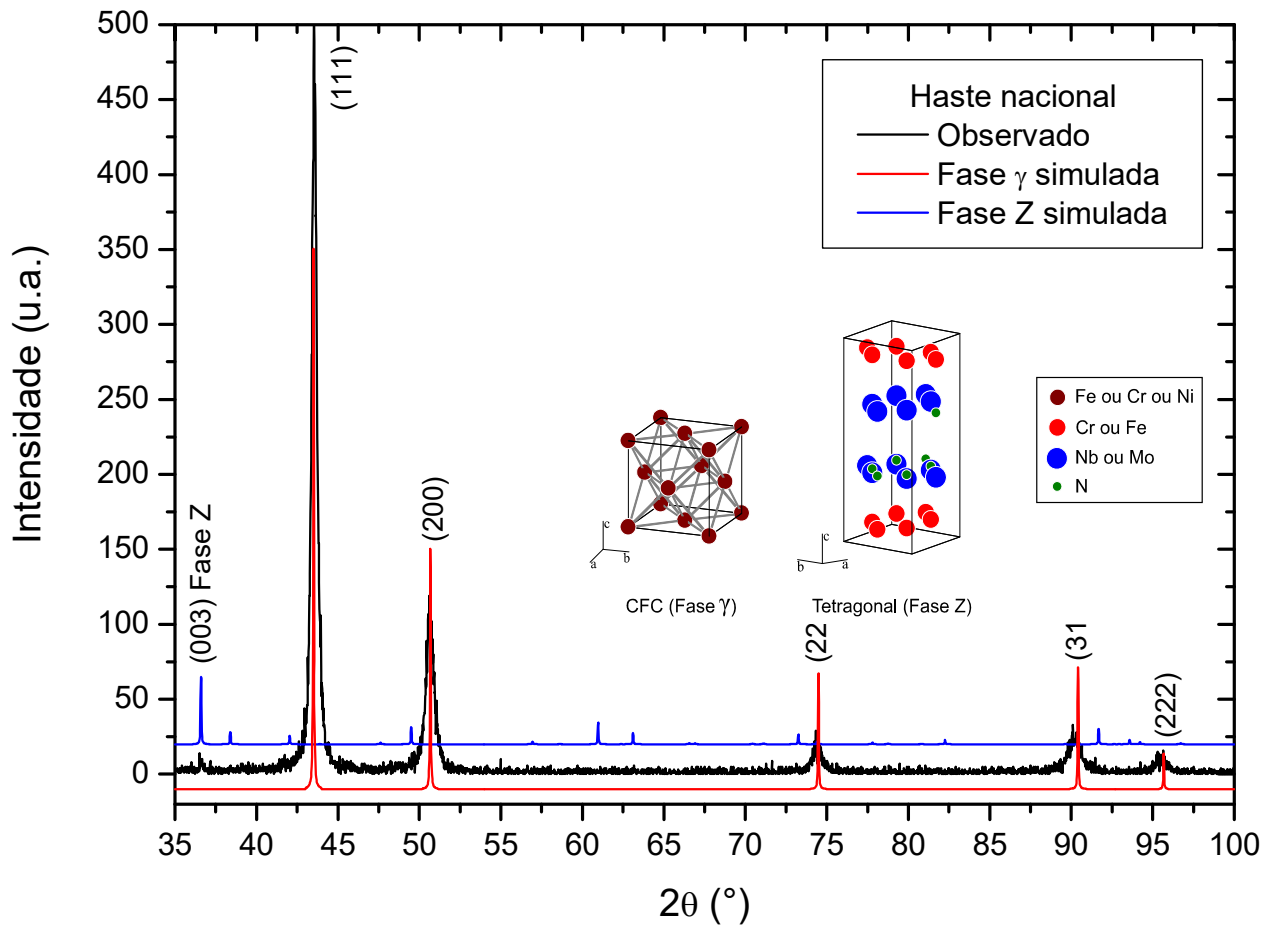

Figura 4. Fases presentes na haste femoral nacional: comparação do difratograma experimental com os difratogramas simulados usando dados cristalográficos da literatura. 


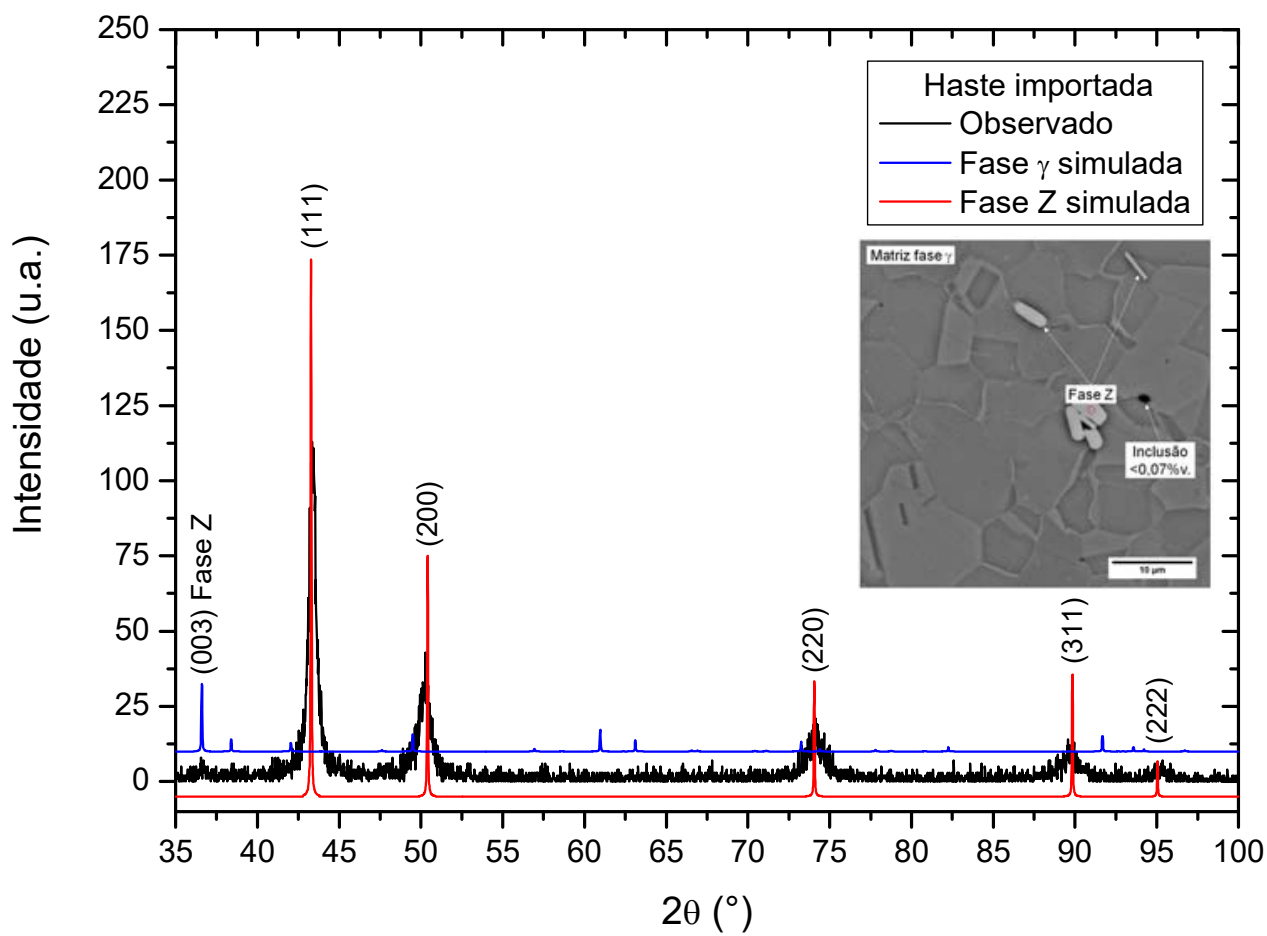

Figura 5. Identificação das fases presentes na haste femoral importada pela comparação do difratograma experimental com os difratogramas simulados usando dados da literatura.

A qualidade dos ajustes dos picos de difração da fase matriz $\gamma$ pelo Método de Rietveld foi similar nas duas hastes. Não foi possível realizar o refinamento da fase $Z$ devido à baixa intensidade dos picos desta fase. A Figura 6 mostra o refinamento para a haste nacional, e a Tabela 3 apresenta os dados cristalográficos obtidos no refinamento.

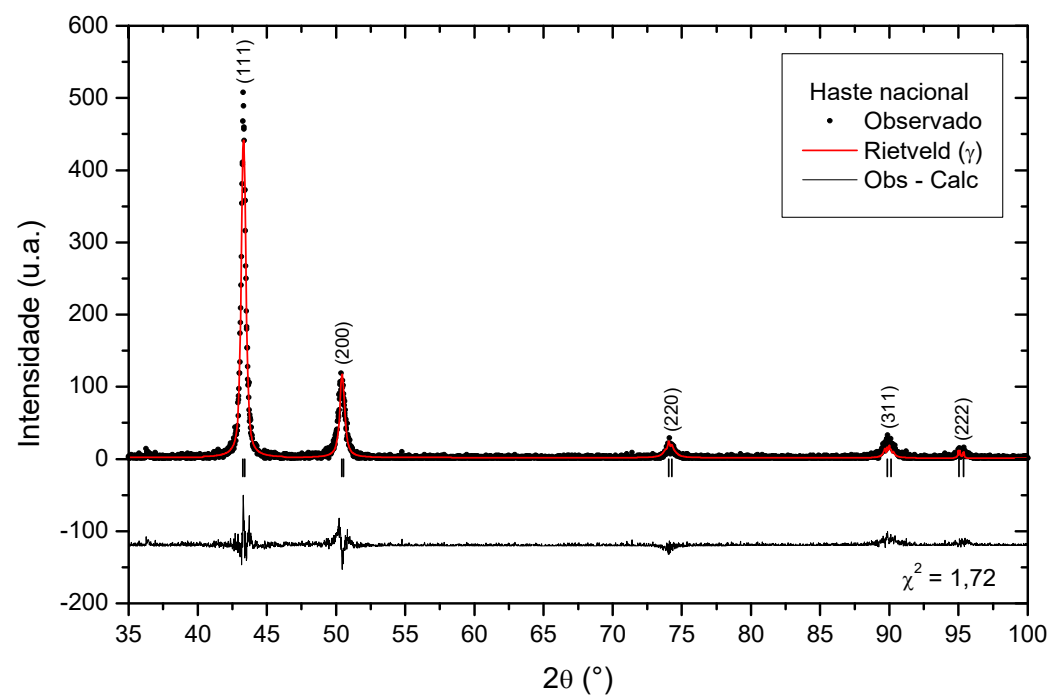

Figura 6. Refinamento de Rietveld da austenita (fase $\gamma$ ). 
Tabela 3. Dados cristalográficos obtidos a partir das estruturas refinadas.

\begin{tabular}{ccccc}
\hline $\begin{array}{c}\text { Haste } \\
\text { femoral }\end{array}$ & $\begin{array}{c}\text { Fração } \\
\text { volumétrica } \\
(\%)\end{array}$ & $\begin{array}{c}\text { Fase } \gamma \text { (CFC) } \\
\text { de rede }(\mathbf{n m})\end{array}$ & $\begin{array}{c}\text { Fase Z (Tetragonal) } \\
\text { volumétrica } \\
(\%)\end{array}$ & $\begin{array}{c}\text { Parâmetro } \\
\text { de rede (nm) }\end{array}$ \\
\hline Nacional & $99,81 \pm 0,06$ & $a=0,3625 \pm 0,0005$ & $0,19 \pm 0,06$ & $a=0,3037$ \\
Importada & $99,85 \pm 0,05$ & $a=0,365 \pm 0,004$ & $0,15 \pm 0,05$ & $c=0,7365$ \\
\hline
\end{tabular}

A quantidade de precipitados da fase $Z$ foi baixa $(\sim 0,2 \% \mathrm{v}$.) e praticamente igual nas duas hastes, indicando que a composição e a microestrutura resultantes estão adequadas. Nas Tabelas 4 e 5 são apresentadas as composições químicas das fases presentes obtidas no EDS. A austenita exibe composição similar nas duas hastes e valores muito próximos da composição global do aço. A fase $Z$ exibiu teores mais elevados de $\mathrm{Nb}, \mathrm{Mo}, \mathrm{Cr}$ e $\mathrm{N}$ nas duas hastes, o que está coerente com os resultados da difração de raios $X$. Os teores dos elementos na fase $Z$ também foram muito próximos nas duas hastes. O maior teor de Fe observado na fase $Z$ da haste importada em relação à haste nacional pode estar associado à contribuição da composição da matriz, devido ao pequeno tamanho das partículas em relação ao diâmetro do feixe de elétrons do MEV.

Durante a análise também foram observadas algumas microinclusões globulares presentes na matriz das duas hastes. Uma destas inclusões é mostrada na micrografia inserida no gráfico da Figura 5. A análise química por EDS (Tabelas $4 \mathrm{e}$ 5) revelou diferenças entre as inclusões presentes nas duas hastes. Enquanto na haste importada as inclusões são ricas em Al, na haste nacional as inclusões são ricas em $\mathrm{Mg}$, provavelmente, estas são alumina e espinélio de magnésio, respectivamente. Inclusões de $\mathrm{Al}_{2} \mathrm{O}_{3}$ geralmente são resultantes da desoxidação do aço com Al ou da contaminação do aço por partículas dos refratários. Já o $\mathrm{Mg}$ geralmente provém de impurezas no Al desoxidante, da decomposição do refratário ou da escória.

Tabela 4. Resultados da análise química por EDS das fases presentes na haste nacional.

\begin{tabular}{cccc}
\hline Elemento & Fase $\gamma$ (\%peso) & Fase Z (\%peso) & Inclusão (\%peso) \\
\hline $\mathrm{C}$ & - & $6,8 \pm 0,7$ & 6,28 \\
$\mathrm{O}$ & - & $3,3 \pm 0,5$ & - \\
$\mathrm{Mn}$ & $4,3 \pm 0,2$ & $1,5 \pm 0,2$ & - \\
$\mathrm{Ni}$ & $9,4 \pm 0,6$ & $1,6 \pm 0,2$ & 1,57 \\
$\mathrm{Cr}$ & $20,8 \pm 0,5$ & $\mathbf{2 7 , 5} \pm \mathbf{0 , 7}$ & 13,94 \\
$\mathrm{Mo}$ & $2,3 \pm 0,2$ & $\mathbf{8 , 4} \pm \mathbf{0 , 6}$ & - \\
$\mathrm{Nb}$ & 0,5 & $\mathbf{2 8} \pm \mathbf{1}$ & 13,6 \\
$\mathrm{Al}$ & - & - & 3,14 \\
$\mathrm{Mg}$ & - & - & $\mathbf{3 8 , 5 8}$ \\
$\mathrm{Ca}$ & - & - & 0,66 \\
$\mathrm{~N}$ & 1,2 & $7,0 \pm 0,3$ & - \\
$\mathrm{Fe}$ & $61 \pm 2$ & $\mathbf{1 6} \pm \mathbf{2}$ & 15,26 \\
$\mathrm{Ti}$ & - & - & 5,6 \\
$\mathrm{~V}$ & - & - & 1,34 \\
\hline
\end{tabular}


Tabela 5. Resultados da análise química por EDS das fases presentes na haste importada.

\begin{tabular}{cccc}
\hline Elemento & Fase $\gamma$ (\%peso) & Fase Z (\%peso) & Inclusão (\%peso) \\
\hline $\mathrm{C}$ & - & $5,4 \pm 0,7$ & $6 \pm 0,1$ \\
$\mathrm{O}$ & - & $3 \pm 1$ & $9 \pm 3$ \\
$\mathrm{Mn}$ & 4,09 & $1,8 \pm 0,4$ & $3,0 \pm 0,6$ \\
$\mathrm{Ni}$ & $9,6 \pm 0,3$ & $3 \pm 1$ & $3,6 \pm 0,5$ \\
$\mathrm{Cr}$ & $19 \pm 2$ & $\mathbf{2 6} \pm \mathbf{3}$ & $11 \pm 2$ \\
$\mathrm{Mo}$ & $2,62 \pm 0,04$ & $\mathbf{8} \pm \mathbf{1}$ & $1,6 \pm 0,4$ \\
$\mathrm{Nb}$ & $0,7 \pm 0,4$ & $\mathbf{2 6} \pm \mathbf{5}$ & - \\
$\mathrm{Al}$ & - & - & $\mathbf{3 8 , 6} \pm \mathbf{9 , 3}$ \\
$\mathrm{Ca}$ & - & - & $0,7 \pm 0,5$ \\
$\mathrm{~N}$ & 1,7 & $7 \pm 2$ & 3,3 \\
$\mathrm{Fe}$ & 61,6 & $\mathbf{2 1} \pm \mathbf{1 0}$ & $28,6 \pm 6$ \\
\hline
\end{tabular}

A Tabela 6 apresenta os teores médios de inclusão observados na seção longitudinal da parte central (região $\mathrm{Cl}$ ) das hastes nacional e importada. Foram observadas apenas microinclusões do tipo D (óxidos globulares) e os teores desta inclusão ficaram abaixo dos limites máximos estabelecidos pela norma ABNT NBR ISO 5832-9, os quais são mostrados nas primeiras colunas da Tabela 6 . A haste importada exibiu menor teor médio de inclusões quando comparado à haste nacional.

Tabela 6. Teor de inclusões e valores limites determinados pela norma ABNT NBR ISO 5832-9.

\begin{tabular}{ccccccc}
\hline & \multicolumn{2}{c}{ ABNT NBR ISO 5832-9 } & \multicolumn{2}{c}{ Nacional } & \multicolumn{2}{c}{ Importada } \\
$\begin{array}{c}\text { Tipo de } \\
\text { Inclusão }\end{array}$ & $\begin{array}{c}\text { Série } \\
\text { Fina }\end{array}$ & Série & Série & Série & Série & Série \\
\hline $\begin{array}{c}\text { G } \\
\text { (Óxidos } \\
\text { Globulares) }\end{array}$ & 2,5 & 1,5 & Fina & Grossa & Gina & Grossa \\
\hline
\end{tabular}

As proporções atômicas entre $\mathrm{Cr}$ e $\mathrm{Fe}$ e entre $\mathrm{Nb}$ e Mo encontradas na fase $\mathrm{Z}$ das hastes foram $\mathrm{Cr} / \mathrm{Fe}=0,6 / 0,4 \mathrm{e} \mathrm{Nb} / \mathrm{Mo}=0,8 / 0,2$, um pouco diferente das proporções de $\mathrm{Cr} / \mathrm{Fe}=0,8 / 0,2$ e $\mathrm{Nb} / \mathrm{Mo}=0,9 /$ 0,1 reportadas por Danielsen e Hald [8]. A Figura 7 exibe o mapa de distribuição dos elementos numa região contendo algumas partículas da fase $\mathrm{Z}$. O mapa confirma o maior teor de $\mathrm{Nb}$ e Mo e o menor teor de $\mathrm{Fe}$ desta fase em comparação com a matriz austenítica. Já o $\mathrm{Cr}$ exibiu concentração mais próxima da matriz.

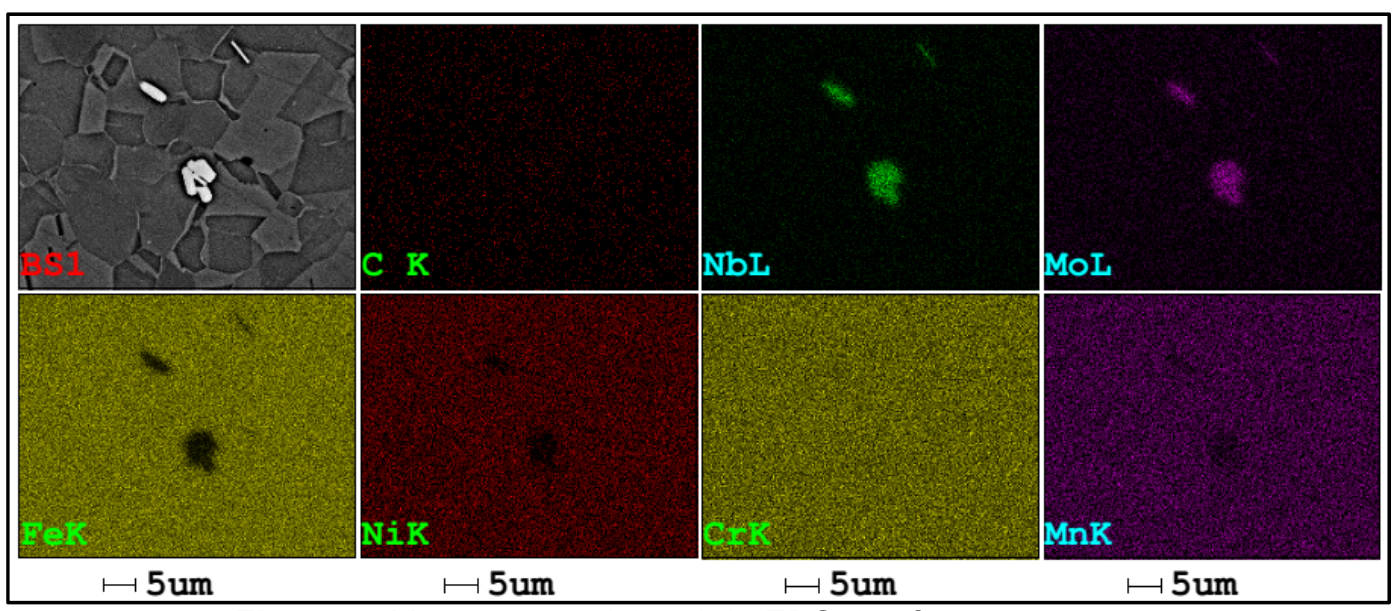

Figura 7. Mapa composicional de EDS das fases presentes. 


\subsection{Tamanho de grão e dureza Vickers}

Para a comparação do tamanho de grão ao longo de cada haste, a Figura 8 mostra as microestruturas nas regiões $A, B, C$ e $D$ e na Tabela 7 são apresentados os valores de tamanho de grão ASTM e a dureza Vickers de cada região.

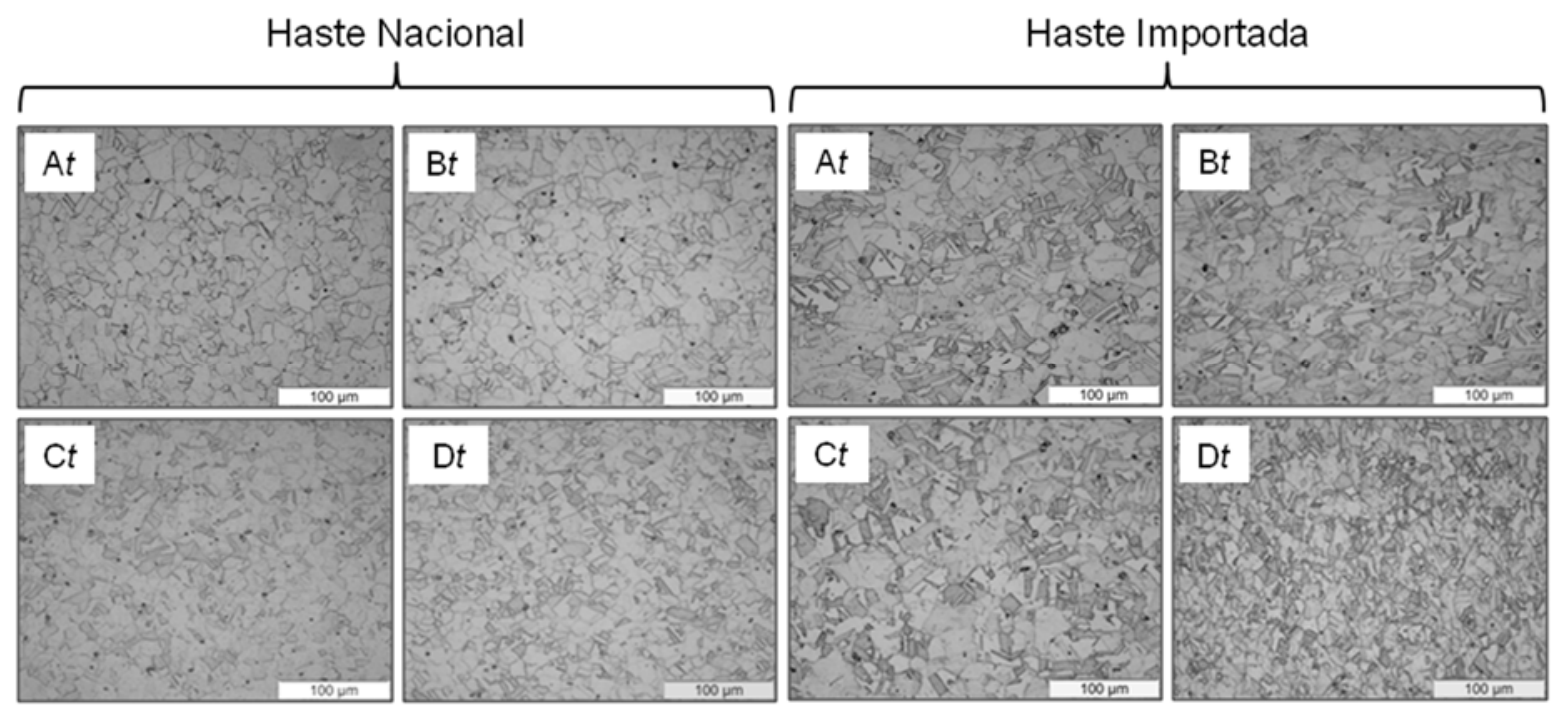

Figura 8. Imagens de microscópio óptico da parte central das seções transversais (regiões A-D) das hastes nacional e importada.

Tabela 7. Tamanho médio de grão e dureza Vickers das hastes nacional e importada.

\begin{tabular}{cccccc}
\hline Haste & $\mathbf{N}^{\circ}$ ASTM & At & B $\boldsymbol{c}$ & $\mathbf{C t}$ & D $\boldsymbol{t}$ \\
\hline \multirow{2}{*}{ Nacional } & $\mathrm{N}^{\circ}$ ASTM & $7,4 \pm 0,6$ & $8,3 \pm 0,2$ & $8,5 \pm 0,4$ & $9,0 \pm 0,6$ \\
& Dureza (HV) & $233 \pm 7$ & $237 \pm 5$ & $238 \pm 8$ & $244 \pm 10$ \\
\multirow{3}{*}{ Importada } & $\mathrm{N}^{\circ}$ ASTM & $8,9 \pm 0,5$ & $8,6 \pm 0,8$ & $8,6 \pm 0,3$ & $9,4 \pm 0,2$ \\
& Dureza (HV) & $342 \pm 22$ & $339 \pm 26$ & $349 \pm 34$ & $362 \pm 37$ \\
\hline
\end{tabular}

O tamanho de grão é aproximadamente homogêneo ao longo do corpo das duas hastes. A norma ABNT NBR ISO 5832-9 estabelece que o menor número ASTM de tamanho de grão deve ser igual a 4, correspondendo a um tamanho de grão máximo de aproximadamente $80 \mu \mathrm{m}$, critério que foi atendido pelas duas hastes. De fato, observando-se a variação do tamanho de grão da região $A$ até $D$ da haste nacional, percebe-se uma redução do tamanho de grão (aumento do $\mathrm{N}^{\circ}$ ASTM) e, consequentemente, um aumento da dureza. A haste importada apresenta tendência similar, porém, neste caso é a região $B$ que exibe o maior tamanho de grão e menor dureza. Embora as hastes tenham apresentado tamanhos de grão praticamente iguais nas regiões $\mathrm{B}, \mathrm{C}$ e D, a dureza Vickers foi $32 \%$ superior na haste importada, sendo este fato atribuído principalmente ao maior teor de $\mathrm{N}$ nesta haste $(0,42 \% \mathrm{p}$.) em comparação com a haste nacional $(0,33 \%$ p.). O nitrogênio tem a capacidade de promover o endurecimento da austenita por solução sólida intersticial [1]. Entretanto, outros elementos também podem estar contribuindo para esta dureza superior. 


\section{CONCLUSÃO}

Tanto a haste femoral nacional como a importada atenderam os requisitos de composição química, tamanho de grão e teor de inclusões da norma ABNT NBR ISO 5832-9.

A haste nacional apresentou microestrutura similar à haste importada, bem como o tamanho de grão da austenita. Algumas inclusões também foram observadas na matriz das duas hastes, sendo as inclusões da haste importada rica em $\mathrm{Al}$ e as inclusões da haste nacional ricas em Mg. Porém, a dureza Vickers foi maior na haste importada devido às diferenças na composição química do aço, principalmente no que se refere ao elemento nitrogênio em quantidade superior na haste importada.

\section{Agradecimentos}

Este trabalho foi financiado pelo Fundo Nacional da Saúde, auxílio $\mathrm{n}^{\circ}$ 25000.162216/2013-11TC 116/2013. O trabalho também recebeu apoio financeiro complementar da FAPERJ, FAPESC e CNPq. Os autores são gratos a Robson Centeno, Rafael Vinhosa, Tatiana Barros, Wellington Gilbert, Alex de Aguiar, Weber Friederichs, Rachel Pereira e Olivia Cypreste Pereira pela realização dos ensaios.

\section{REFERÊNCIAS}

1 Giordani EJ, Ferreira I, Balancin O. Propriedades mecânicas e de corrosão de dois aços inoxidáveis austeníticos utilizados na fabricação de implantes ortopédicos. Rem Rev Esc Minas. 2007;60(1):55-62.

2 Giordani EJ, Guimarães VA, Pinto TB, Ferreira I. Effect of precipitates on the corrosionfatigue crack initiation of ISO 5832-9 stainless steel biomaterial. Int J Fatigue [Internet]. 2004 Oct;26(10):1129-36. Available from: http://linkinghub.elsevier.com/retrieve/pii/S014211230400057X

3 Ornhagen C, Nilsson JO, Vannevik H. Characterization of a nitrogen-rich austenitic stainless steel used for osteosynthesis devices. J Biomed Mater Res [Internet]. 1996;31(1):97-103. Available from: http://www.ncbi.nlm.nih.gov/pubmed/8731154

4 Giordano EJ, Alonso-Falleiros N, Ferreira I, Balancin O. Electrochemical behavior of two austenitic stainless steel biomaterials. Rem Rev Esc Minas [Internet]. 2010 Mar;63(1):159-66. Available from: http://www.scielo.br/scielo.php?script=sci_arttext\&pid=S037044672010000100027\&lng=en\&nrm=iso\&tlng=en

5 Grabke HJ. High Nitrogen Steels. The Role of Nitrogen in the Corrosion of Iron and Steels. ISIJ Int [Internet]. 1996;36(7):777-86. Available from: http://www.journalarchive.jst.go.jp/jnlpdf.php?cdjournal=isijinternational 1989\&cdvol=36\& noissue $=7$ \&startpage $=777$ \&lang=en\&from $=$ jnlabstract

6 Sobrinho JHOS, Rodrigues SF, Silva ES, Reis GS, Sousa RC, Leal VS, et al. Plastic Instability in ISO 5832-9 High-nitrogen Austenitic Stainless Steel. ISIJ Int [Internet]. 2015;55(3):670-8. Available from:

https://www.jstage.jst.go.jp/article/isijinternational/55/3/55_670/_article

7 Fonseca e Silva E, Oliveira LF. Evaluation of the corrosion resistance of stainless steel ISO 5832-9 and F138 implants removed from patients. Quim Nova. CAIXA POSTAL 26037, 05599-970 SAO PAULO, BRAZIL: SOC BRASILEIRA QUIMICA; 2012;35(1):59-65.

8 Danielsen HK, Hald J. A thermodynamic model of the Z-phase $\mathrm{Cr}(\mathrm{V}, \mathrm{Nb}) \mathrm{N}$. Calphad [Internet]. 2007;31(4):505-14. Available from:

http://linkinghub.elsevier.com/retrieve/pii/S0364591607000417 